\title{
SYSTEM IDENTIFICATION USING FRACTIONAL MODELS: STATE OF THE ART
}

\author{
Rachid Malti, Stéphane Victor, Olivier Nicolas, and Alain Oustaloup \\ IMS, UMR 5218 CNRS - Université Bordeaux 1 \\ 351 cours de la Libération, F 33405 Talence cedex, France \\ \{rachid.malti, stephane.victor, olivier.nicolas, alain.oustaloup\}@ims-bordeaux.fr
}

\begin{abstract}
This paper presents a state of the art of actual achievements in time-domain system identification using fractional models. It starts with some general aspects on time and frequency-domain representations, time-domain simulation, and stability of fractional models. Then, an overview on system identification methods using fractional models is presented. Both equation-error and output-error-based models are detailed. In the former models, prior knowledge is generally used to fix differentiation orders; model coefficients are estimated using least squares. The latter models allow simultaneous estimation of model's coefficients and differentiation orders, using non linear programming. A real thermal example is identified using a fractional model and compared to a rational one.
\end{abstract}

\section{Introduction}

Although fractional (non integer) operators remained for a long time purely a mathematical concept, the rise of digital computers offered an easy way for simulating numerically non integer integro-differentiation of mathematical functions.

The last two decades have witnessed considerable development in the use of fractional differentiation in various fields. Fractional differentiation is now an important tool for the international scientific and industrial communities. The use of fractional differentiation models in system identification was initiated in the late nineties and the beginning of this century [1-4].

Fractional models are now enough mature and are widely used in representing thermal diffusive phenomena [5], electrochemical diffusion [6], and in modeling viscoelastic materials.
See the special issue of Signal Processing [7] for other fields of application.

\subsection{Mathematical background}

A fractional mathematical model is based on fractional differential equation:

$$
\begin{aligned}
y(t)+b_{1} \mathbf{D}^{\beta_{1}} y(t) & +\cdots+b_{m_{B}} \mathbf{D}^{\beta_{m_{B}}} y(t)= \\
& a_{0} \mathbf{D}^{\alpha_{0}} u(t)+a_{1} \mathbf{D}^{\alpha_{1}} u(t)+\cdots+a_{m_{A}} \mathbf{D}^{\alpha_{m_{A}}} u(t)
\end{aligned}
$$

where differentiation orders, $\beta_{1}<\beta_{2}<\ldots<\beta_{m_{B}}, \alpha_{0}<\alpha_{1}<$ $\ldots<\alpha_{m_{A}}$, are allowed to be non-integer positive numbers. The concept of differentiation to an arbitrary order (non-integer),

$$
\mathbf{D}^{\gamma} \triangleq\left(\frac{d}{d t}\right)^{\gamma} \quad \forall \gamma \in \mathbb{R}_{+}^{*}
$$

was defined in the $19^{\text {th }}$ century by Riemann and Liouville. The $\gamma$ fractional derivative of $x(t)$ is defined as being an integer derivative of order $\lfloor\gamma\rfloor+1$ ( $\lfloor$.$\rfloor stands for the floor operator) of a non-$ integer integral of order $\gamma-\lfloor\gamma\rfloor[8]$ :

$$
\begin{aligned}
\mathbf{D}^{\gamma} x(t)= & \mathbf{D}^{\lfloor\gamma\rfloor+1}\left(\mathbf{I}^{\lfloor\gamma\rfloor+1-\gamma} x(t)\right) \triangleq \\
& \left(\frac{d}{d t}\right)^{\lfloor\gamma\rfloor+1}\left(\frac{1}{\Gamma(\lfloor\gamma\rfloor+1-\gamma)} \int_{0}^{t} \frac{x(\tau) d \tau}{(t-\tau)^{\gamma-\lfloor\gamma\rfloor}}\right)
\end{aligned}
$$


where $t>0, \forall \gamma \in \mathbb{R}_{+}^{*}$, and the Euler's $\Gamma$ function is defined as:

$$
\Gamma(x)=\int_{0}^{\infty} e^{-t} t^{x-1} d t \quad \forall x \in \mathbb{R}^{*} \backslash\left\{\mathbb{N}^{-}\right\}
$$

A discrete-time definition of fractional derivative was proposed by Grünwald [9], $\forall \gamma \in \mathbb{R}_{+}^{*}$ :

$$
\mathbf{D}^{\gamma} x(t)=\lim _{h \rightarrow 0} \frac{1}{h^{\gamma}} \sum_{k=0}^{\infty}(-1)^{k}\left(\begin{array}{l}
\gamma \\
k
\end{array}\right) x(t-k h)
$$

where Newton's binomial $\left(\begin{array}{l}\gamma \\ k\end{array}\right)$ is generalized to non-integer orders by the use of Euler's $\Gamma$ function:

$$
\left(\begin{array}{l}
\gamma \\
k
\end{array}\right)=\frac{\Gamma(\gamma+1)}{\Gamma(k+1) \Gamma(\gamma-k+1)}
$$

Equation (4) is generally used in time-domain simulations of fractional differentiation. As Newton's binomial $\left(\begin{array}{l}\gamma \\ k\end{array}\right)$ does not converge rapidly to zero with $k$ when $\gamma$ is non integer, the computation of $\mathbf{D}^{\gamma} x(t)$ depends on all values of $x(t)$ between 0 and $t$ (supposing that $x(t)$ is relaxed at $t=0$, i.e. $x(t)=0 \forall t<0$ ). Since fractional derivatives of a function depend on its whole past, fractional operators are known to have long memory behavior.

A more concise algebraic tool can be used to represent fractional systems: the Laplace transform [10]

$$
\mathscr{L}\left\{\mathbf{D}^{\gamma} x(t)\right\}=s^{\gamma} X(s) \quad \text { if } x(t)=0 \forall t<0
$$

This property allows to write the fractional differential equation (1), provided $u(t)$ and $y(t)$ are relaxed at $t=0$, in a transfer function form:

$$
F(s)=\frac{\sum_{i=0}^{m_{A}} a_{i} s^{\alpha_{i}}}{1+\sum_{j=1}^{m_{B}} b_{j} s^{\beta_{j}}}
$$

where $\left(a_{i}, b_{j}\right) \in \mathbb{R}^{2}, \quad\left(\alpha_{i}, \beta_{j}\right) \in \mathbb{R}_{+}^{2}, \forall i=0,1, \ldots, m_{A}, \forall j=$ $1,2, \ldots, m_{B}$.

A transfer function $F(s)$ is commensurable of order $\gamma$ iff it can be written as $F(s)=S\left(s^{\gamma}\right)$, where $S=\frac{T}{R}$ is a rational function with $T$ and $R$ two co-prime polynomials. Moreover, the commensurable order $\gamma$ is the biggest number satisfying the aforementioned condition.
In other words, the commensurable order $\gamma$ is defined as the biggest real number such that all differentiation orders are integer multiples of $\gamma$. Taking as an example $F(s)$ defined in (6), assuming that $F(s)$ is commensurable of order $\gamma$, and using $F(s)=S\left(s^{\gamma}\right)$, one can write:

$$
S(s)=\frac{T(s)}{R(s)}=\frac{\sum_{m=0}^{m_{A}} a_{m} s^{\frac{\alpha_{m}}{\gamma}}}{1+\sum_{m=1}^{m_{B}} b_{m} s^{\frac{\beta_{m}}{\gamma}}}
$$

where all powers of $s$ in (7) are integers.

A modal form transfer function can then be obtained, provided (6) is strictly proper:

$$
F(s)=\sum_{k=1}^{N} \sum_{q=1}^{v_{k}} \frac{A_{k, q}}{\left(s^{\gamma}-s_{k}\right)^{q}}
$$

where $s_{k}, k=1, \cdots, N$ are known as the $s^{\gamma}$-poles of integer multiplicity $v_{k}$. The elementary transfer function $\frac{A_{k, l}}{\left(s^{\gamma}-s_{k}\right)^{q}}$ is known as a mode of order $\gamma$ and of multiplicity $q$.

\subsection{State space representation}

If the system defined by the fractional differential equation (1) is commensurable of order $\gamma$, then (1) can be rewritten in a "fractional" (also called "generalized" or "macro") state-space representation [11-13]:

$$
\left\{\begin{array}{l}
\mathbf{D}^{\gamma} x(t)=\mathbf{A} x(t)+\mathbf{B} u(t) \\
y(t)=\mathbf{C} x(t)+\mathbf{E} u(t)
\end{array}\right.
$$

This representation suggests the possibility of developing subspace identification methods.

\subsection{Stability theorem}

Here is a modified version of Matignon's [14] theorem. A commensurable $\gamma$-order transfer function $F(s)=S\left(s^{\gamma}\right)=$ $\frac{T\left(s^{\gamma}\right)}{R\left(s^{\gamma}\right)}$, where $T$ and $R$ are two coprime polynomials, is BIBO stable iff

$$
0<\gamma<2
$$

and for every $s \in \mathbb{C}$ such that $R(s)=0$

$$
|\arg (s)|<\gamma \frac{\pi}{2}
$$




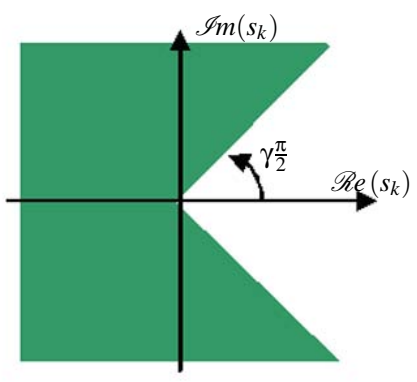

Figure 1. Stability region. A system is stable iff all its $s^{\gamma}$-poles are inside the greyed region

The stability region suggested by this theorem and plotted in figure (1) tends to the whole $s$-plane when $\gamma$ tends to 0 , corresponds to the Rooth-Hurwitz stability when $\gamma=1$, and tends to the negative real axis when $\gamma$ tends to 2 . An additional condition is however required for a system to belong to $\mathrm{H}_{2}\left(\mathbb{C}^{+}\right)$.

\section{$1.4 H_{2}$ norm of fractional transfer functions}

Contrary to rational systems, the stability condition does not guarantee that a fractional transfer function belongs to $H_{2}\left(\mathbb{C}^{+}\right)$. The $H_{2}$ norm of fractional systems is extensively studied by Malti et al. in [15], where the authors showed that a stable fractional transfer function as defined in (6), where conditions (10) and (11) are satisfied, belongs to $H_{2}\left(\mathbb{C}^{+}\right)$iff its relative degree is greater than $\frac{1}{2}$ :

$$
\beta_{m_{B}}-\alpha_{m_{A}}>\frac{1}{2}
$$

This condition suggests that some stable fractional systems not satisfying (12) have infinite impulse response energy. Those systems cannot be represented using fractional orthogonal bases which span $\mathrm{H}_{2}\left(\mathbb{C}^{+}\right)$, as described in [16-18].

\subsection{Equivalence with rational models}

Due to the consideration that real physical systems generally have bandlimited fractional behavior and due to the practical limitations of input and output signals (Shannon's cut-off frequency for the upper band and the spectrum of the input signal for the lower band), fractional operators are usually approximated by high order rational models. As a result, a fractional model and its rational approximation have the same dynamics within a limited frequency band. The most commonly used approximation of $s^{\gamma}$ in the frequency band $\left[\omega_{A}, \omega_{B}\right]$ is the recursive distribution of zeros and poles proposed by Oustaloup [19]. Trigeassou et al. [13] suggested to use an integrator outside the frequency range

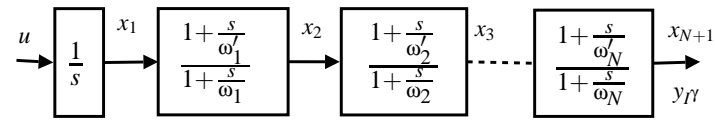

Figure 2. Approximation of a fractional integrator using a rational model

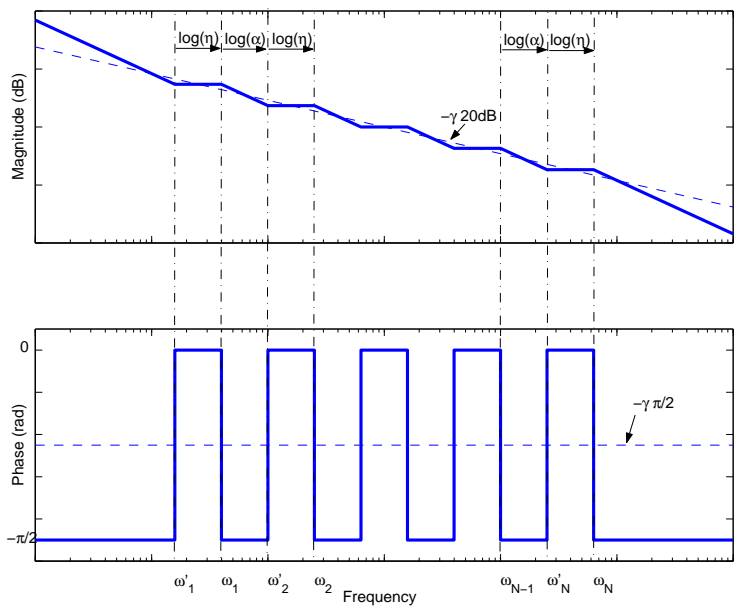

Figure 3. Bode diagrams of a fractional integrator and the asymptotic behavior of its rational approximation

$\left[\omega_{A}, \omega_{B}\right]$ instead of a gain:

$$
s^{-\gamma} \rightarrow s_{\left[\omega_{A}, \omega_{B}\right]}^{-\gamma}=\frac{C_{0}}{s}\left(\frac{1+\frac{s}{\omega_{A}}}{1+\frac{s}{\omega_{B}}}\right)^{1-\gamma} \approx \frac{C_{0}}{s} \prod_{k=1}^{N} \frac{1+\frac{s}{\omega_{k}^{\prime}}}{1+\frac{s}{\omega_{k}}}
$$

where $\omega_{k}=\alpha \omega_{k}^{\prime}, \quad \omega_{k+1}^{\prime}=\eta \omega_{k}^{\prime}$ and

$$
\gamma=1-\frac{\log \alpha}{\log \alpha \eta}
$$

$\alpha$ and $\eta$ are real parameters which depend on the differentiation order $\gamma$. The bigger $N$ the better the approximation of the integrator $s^{-\gamma}$.

The obtained integrator has the following state space representation:

$$
\begin{aligned}
\dot{\mathbf{x}} & =\mathbf{A} \mathbf{x}+\mathbf{B} u \\
y_{I \gamma} & =\mathbf{C x}
\end{aligned}
$$


where $y_{I^{\gamma}}$ is the derivative of the input $u$ (see figure (2)),

$$
A=\left[\begin{array}{ccccc}
1 & 0 & \cdots & \cdots & 0 \\
-\alpha & 1 & & & \vdots \\
0 & -\alpha & 1 & & \vdots \\
\vdots & & \ddots & \ddots & 0 \\
0 & \cdots & 0 & -\alpha & 1
\end{array}\right]^{-1}\left[\begin{array}{ccccc}
0 & 0 & \cdots & \cdots & 0 \\
\omega_{1} & -\omega_{1} & & & \vdots \\
0 & \omega_{2} & -\omega_{2} & & \vdots \\
\vdots & & \ddots & \ddots & 0 \\
0 & \cdots & 0 & \omega_{N} & -\omega_{N}
\end{array}\right]
$$

$$
\mathbf{B}=\left[\begin{array}{ccccc}
1 & 0 & \cdots & \cdots & 0 \\
-\alpha & 1 & & & \vdots \\
0 & -\alpha & 1 & & \vdots \\
\vdots & & \ddots & \ddots & 0 \\
0 & \cdots & 0 & -\alpha & 1
\end{array}\right]^{-1}\left[\begin{array}{c}
C_{0} \\
0 \\
\vdots \\
\vdots \\
0
\end{array}\right]
$$

and

$$
\mathbf{C}=\left[\begin{array}{llll}
0 & \cdots & 0 & 1
\end{array}\right]
$$

\section{System identification}

Frequency-domain system identification using fractional models was initiated by the Ph.D thesis of Le Lay [1]. Timedomain system identification using fractional differentiation models was initiated by the Ph.D theses of Le Lay [1], Lin [2], and Cois [3]. Mainly two classes of models were developed: Equation-error-based models and Output-Error-based models, both of which are presented in this section. Recently Malti et al. [16] synthesized fractional orthogonal bases generalizing Laguerre, Kautz and BOG bases to fractional differentiation orders.

\subsection{Equation-error models}

Equation-error-based models are linear in coefficients. The identified system is assumed to be initially at rest, modeled by (1), and characterized by input/output coefficient's vector:

$$
\theta=\left[a_{0} \ldots a_{m_{A}} b_{1} \ldots b_{m_{B}}\right]^{\mathbf{T}}
$$

Prior knowledge is generally used to fix the differentiation orders $\alpha_{0}, \ldots, \alpha_{m_{A}}, \beta_{1}, \ldots, \beta_{m_{B}}$. Usually, a commensurable order $\gamma$ is chosen and then all its multiples fixed up to a given order, say $\beta_{m_{B}}$. The order $\alpha_{m_{A}}$ is generally set to $\beta_{m_{B}}-\gamma$ for strictly proper systems.

$$
F(s)=\frac{\sum_{k=0}^{\frac{\alpha_{m_{A}}}{\gamma}} a_{k} s^{k \gamma}}{1+\sum_{j=1}^{\frac{\beta_{m_{B}}}{\gamma}} b_{j} s^{j \gamma}}
$$

Consider observed data $u(t)$ and $y^{*}(t)=y(t)+p(t)$, where $p(t)$ is a perturbation signal, collected at regular samples: $k_{0} T_{s},\left(k_{0}+\right.$ 1) $T_{s}, \ldots,\left(k_{0}+K-1\right) T_{s}$. The most basic estimation method consists of computing fractional derivatives of input/output signals from sampled data by applying (4). The output can be written in a regression form:

$$
y(t)=\phi^{*}(t) \theta
$$

where parameters and regression vectors are respectively given by (16) and:

$$
\phi^{*}(t)=\left[\mathbf{D}^{\alpha_{0}} u(t) \cdots \mathbf{D}^{\alpha_{m_{A}}} u(t)-\mathbf{D}^{\beta_{1}} y^{*}(t) \cdots-\mathbf{D}^{\beta_{m_{B}}} y^{*}(t)\right]
$$

Estimated parameters vector $\hat{\theta}$ of $\theta$ is obtained by minimizing the quadratic norm of the error:

$$
J(\hat{\theta})=\mathbf{E}^{\mathrm{T}} \mathbf{E}
$$

where:

$$
\mathbf{E}=\left[\begin{array}{ll}
\varepsilon\left(k_{0} T_{\mathrm{s}}\right) \varepsilon\left(\left(k_{0}+1\right) T_{\mathrm{s}}\right) & \cdots \varepsilon\left(\left(k_{0}+K-1\right) T_{\mathrm{s}}\right)
\end{array}\right]^{\mathrm{T}}
$$

and

$$
\varepsilon(t)=y^{*}(t)-\phi^{*}(t) \hat{\theta}
$$

The minimum of $J$ is given by the classical least squares:

$$
\hat{\theta}_{\mathbf{o p t}}=\left(\Phi^{* \mathrm{~T}} \Phi^{*}\right)^{-1} \Phi^{* \mathrm{~T}} \mathbf{Y}^{*}
$$

where:

$$
\begin{aligned}
& \Phi^{*}=\left[\begin{array}{lll}
\phi^{* T}\left(k_{0} T_{s}\right) & \phi^{* T}\left(\left(k_{0}+1\right) T_{s}\right) & \ldots
\end{array}\right. \\
& \left.\phi^{* T}\left(\left(k_{0}+K-1\right) T_{s}\right)\right]^{\mathrm{T}}
\end{aligned}
$$


As in the integer case, fractional differentiation of noisy signals amplifies the noise. Hence, a linear transformation (lowpass filter) can be applied to (18) so as to obtain a linear continuous regression of filtered input, $u_{f}(t)$, and output, $y_{f}^{*}(t)$, signals:

$$
y_{f}(t)=\phi_{f}^{*}(t) \theta
$$

where

$\phi_{f}^{*}(t)=\left[\mathbf{D}^{\alpha_{0}} u_{f}(t) \cdots \mathbf{D}^{\alpha_{m_{A}}} u_{f}(t) \quad-\mathbf{D}^{\beta_{1}} y_{f}^{*}(t) \cdots-\mathbf{D}^{\beta_{m_{B}}} y_{f}^{*}(t)\right]$

The filter is generally chosen to be causal, stationary, and lowpass. Among the possible filters the linear integral filter can be used:

$$
H(s)=\left(\frac{1}{s}\right)^{L}
$$

where the order $L$ is generally chosen as the highest differentiation order in (1), i.e. $L=\beta_{m_{B}}$. In this case, the differential equation (1) is rewritten as an integral equation:

$$
\begin{aligned}
& \mathbf{D}^{-\beta_{m_{B}}} y(t)+b_{1} \mathbf{D}^{\beta_{1}-\beta_{m_{B}}} y(t)+\cdots+b_{m_{B}} y(t)= \\
& a_{0} \mathbf{D}^{\alpha_{0}-\beta_{m_{B}}} u(t)+\cdots+a_{m_{A}} \mathbf{D}^{\alpha_{m_{A}-\beta_{m_{B}}}} u(t)
\end{aligned}
$$

where all superscripts of $\mathbf{D}$ are negative which stands for integration operator.

Although this method avoids differentiation of noisy signals, it however integrates noise, producing wrong steady-state and low dynamics estimations. Among the possible filters, Cois et al. [20] extend the concept of State Variable Filters (SVF) [21] to fractional differentiation systems. They propose to use the following fractional filter:

$$
H(s)=\frac{A}{\alpha_{0}+\alpha_{1} s^{\gamma}+\ldots+\alpha_{N_{f}-1} s^{\gamma\left(N_{f}-1\right)}+s^{\gamma N_{f}}}
$$

$\gamma N_{f}$ is filter's order. The design must respect the following specifications:

$N_{f}>\max \left(\beta_{m_{B}}, \alpha_{m_{A}}\right)$

Coefficients $\alpha_{0}, \alpha_{1}, \ldots, \alpha_{N_{f}-1}$ must be chosen such that $H(s)$ is stable.

A particular choice of SVF, proposed by Cois et al. [20], is the fractional Poisson's filter:

$$
\begin{aligned}
& H(s)=\frac{1}{\left(\left(\frac{s}{\omega_{f}}\right)^{\gamma}+1\right)^{N_{f}}}= \\
& \frac{\omega_{f}^{\gamma N_{f}}}{s^{\gamma N_{f}}+\left(\begin{array}{l}
N_{f} \\
1
\end{array}\right) \omega_{f}^{\gamma} s^{\gamma\left(N_{f}-1\right)}+\ldots+\left(\begin{array}{l}
N_{f} \\
N_{f}-1
\end{array}\right) \omega_{f}^{\gamma\left(N_{f}-1\right)} s^{\gamma}+\omega_{f}^{\gamma N_{f}}}
\end{aligned}
$$

which is simply an extension of the rational Poisson's filter to fractional differentiation orders. Frequency $\omega_{f}$ is fixed by the user according to the frequency characteristics of the system to be identified (close to the highest corner frequency). The state vector, composed of fractional derivatives of filtered input or output signals, is defined by:

$$
x_{f}=\left[\begin{array}{r}
\mathbf{D}^{\left(N_{f}-1\right) \gamma_{z_{f}}(t),}, \mathbf{D}^{\left(N_{f}-2\right) \gamma_{z_{f}}(t),} \\
\ldots, \mathbf{D}^{\gamma_{z_{f}}}(t), z_{f}(t)
\end{array}\right]^{\mathrm{T}}
$$

where $z_{f}$ denotes either $u_{f}$ or $y_{f}$. The fractional state space representation of the filter is given by:

$$
\mathbf{D}^{\gamma} x_{f}(t)=A_{f} x_{f}(t)+B_{f} z_{f}(t)
$$

where $A_{f}=-$

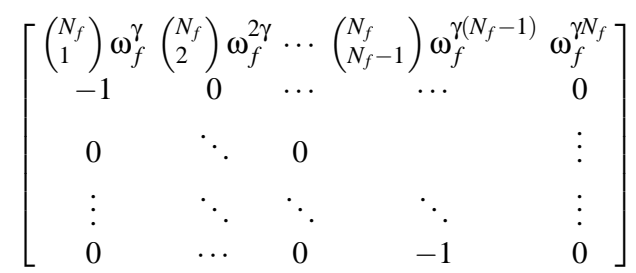

and

$$
B_{f}=\left[\begin{array}{llll}
\omega_{f}^{\gamma N_{f}} & 0 & \ldots & 0
\end{array}\right]^{T}
$$

Each state represents the derivative of a given order of input or output signals (see figure (4)). Fractional Poisson's filters are simulated using (4)

The estimated parameters vector $\hat{\theta}$ of $\theta$ is now obtained by minimizing the quadratic norm of the filtered equation error:

$$
J(\hat{\theta})=\mathbf{E}_{\mathbf{f}}^{\mathrm{T}} \mathbf{E}_{\mathbf{f}}
$$




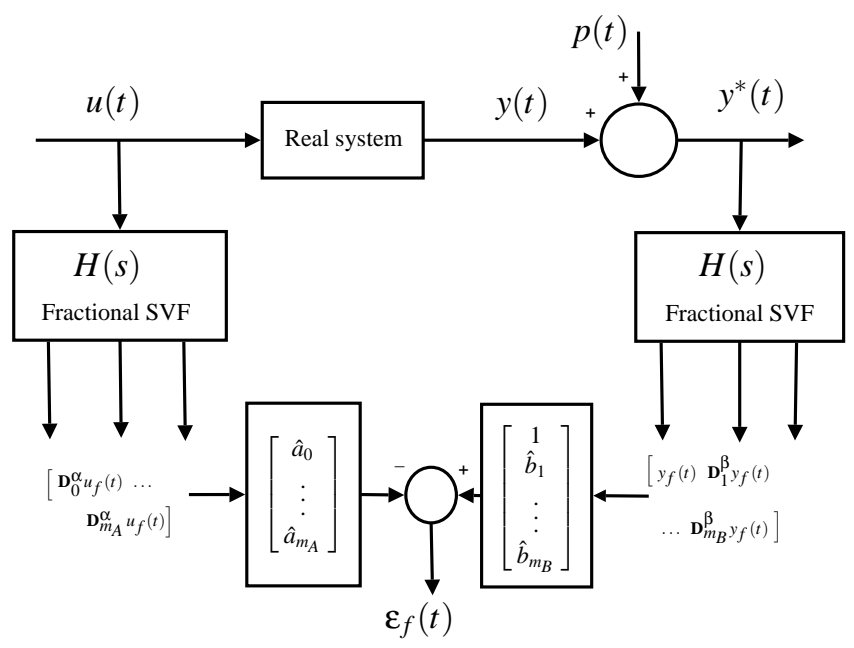

Figure 4. Fractional state variable filters

where:

$$
\mathbf{E}_{\mathbf{f}}=\left[\begin{array}{lll}
\varepsilon_{f}\left(k_{0} T_{\mathrm{s}}\right) \varepsilon_{f}\left(\left(k_{0}+1\right) T_{\mathrm{s}}\right) & \cdots \varepsilon_{f}\left(\left(k_{0}+K-1\right) T_{\mathrm{s}}\right)
\end{array}\right]^{\mathrm{T}}
$$

and

$$
\varepsilon_{f}(t)=y_{f}^{*}(t)-\phi_{f}^{*}(t) \hat{\theta},
$$

$\phi_{f}^{*}$ being defined by (23).

The solution is given by the classical least squares:

$$
\hat{\theta}=\left(\Phi_{\mathrm{f}}^{\mathrm{T}} \Phi_{f}\right)^{-1} \Phi_{f}^{\mathrm{T}} \mathbf{Y}_{f}^{*}
$$

where:

$$
\Phi_{f}^{*}=\left[\phi_{f}^{* T}\left(k_{0} T_{s}\right) \phi_{f}^{* T}\left(\left(k_{0}+1\right) T_{s}\right) \ldots \phi_{f}^{* T}\left(\left(k_{0}+K-1\right) T_{s}\right)\right]
$$

As in the classical case, Cois et al. [20] showed that the least squares estimator (30) is biased in presence of noisy output. To eliminate the bias, they propose to use instrumental variable method. Parameters are estimated according to:

$$
\hat{\theta}_{\mathrm{opt}}^{\mathrm{IV}}=\left(\Phi_{f}^{\mathrm{IV}} \Phi_{f}^{*}\right)^{-1} \Phi_{f}{ }^{\mathrm{IV} \mathrm{T}} \mathbf{Y}_{f}^{*}
$$

where $\Phi_{f}^{I V}$ is the regression matrix constituted of derivatives of filtered inputs and derivatives of instrumental variables. The authors also suggest to optimize instruments by an iterative method.

\subsection{Output-error models}

Output-error-based models allow simultaneous estimation of differentiation orders and model parameters. Mainly, three identification methods were proposed in the literature. The first one is based on discrete-time simulation of fractional models. The second one, based on continuous-time simulation of fractional models, assumes that the fractional behavior is present in a limited frequency band. The third one uses fractional orthogonal functions.

\subsubsection{Method based on discrete-time simulation} of fractional models Here, the system to be identified is assumed to be initially at rest, modeled by (6). It is now characterized by input/output vector formed of coefficients and differentiation orders $\theta=\left[a_{0}, \ldots a_{m_{A}}, b_{1}, \ldots b_{m_{B}}, \alpha_{0}, \ldots \alpha_{m_{A}}, \beta_{1}, \ldots \beta_{m_{B}}\right]$.

When the number of parameters in (6) is high, optimization algorithms are ill-conditioned due to the absence of constraints on differentiation orders. One way for introducing a constrained optimization on differentiation orders and, at the same time, limiting the number of parameters consists of optimizing the commensurable order $\gamma$ instead of all differentiation orders. In this case, the fractional transfer function (6) is rewritten in a commensurable form as in (17).

Numerator and denominator orders, respectively $\alpha_{m_{A}}$ and $\beta_{m_{B}}$ (both multiples of $\gamma$ ), are fixed as in classical rational models. Henceforth, the system is entirely characterized by coefficients' vector: $\theta=\left[a_{0}, \ldots, a_{m_{A}}, b_{1}, \ldots, b_{m_{B}}, \gamma\right]$. As far as identification of stable systems is concerned, the commensurable order can be constrained to $] 0,2[$ (see conditions (10) and (11) of the stability theorem).

Considering observed data $u(t)$ and $y^{*}(t)=y(t)+p(t), p(t)$ being an output white noise, the quadratic norm:

$$
J(\hat{\theta})=\sum_{k=k_{0}}^{k_{0}+K-1} \varepsilon^{2}\left(k T_{s}, \hat{\theta}\right)
$$

of output error:

$$
\varepsilon\left(k T_{s}, \hat{\theta}\right)=y^{*}\left(k T_{s}\right)-\hat{y}\left(k T_{s}, \hat{\theta}\right)
$$

is now minimized. Model's output $\hat{y}\left(k T_{s}, \hat{\theta}\right)$ being non linear in $\hat{\theta}$, gradient-based algorithms, such as the Marquardt algorithm [22], are used to estimate $\hat{\theta}$ iteratively:

$$
\hat{\theta}_{i+1}=\hat{\theta}_{i}-\left\{\left[\mathbf{J}_{\theta \theta}^{\prime \prime}+\xi \mathbf{I}\right]^{-1} \mathbf{J}_{\theta}^{\prime}\right\}_{\theta=\hat{\theta}_{i}}
$$




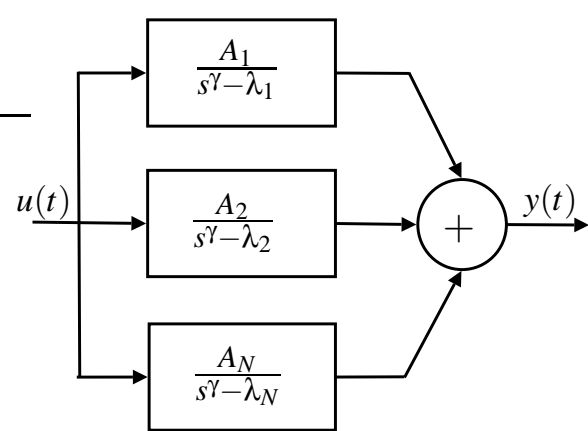

Figure 5. Modal Decomposition

$$
\left\{\begin{array}{l}
\mathbf{J}_{\theta}^{\prime}=-2 \sum_{k=k_{0}}^{k_{0}+K-1} \varepsilon\left(k T_{S}\right) \mathbf{S}\left(k T_{s}, \hat{\theta}\right): \text { gradient } \\
\mathbf{J}_{\theta \theta}^{\prime \prime} \approx 2 \sum_{k=k_{0}}^{k_{0}+K-1} \mathbf{S}\left(k T_{S}, \hat{\theta}\right) \mathbf{S}^{\mathrm{T}}\left(k T_{S}, \hat{\theta}\right): \text { pseudo - hessian } \\
\mathbf{S}\left(k T_{s}, \hat{\theta}\right)=\frac{\partial \hat{y}\left(k T_{s}, \hat{\theta}\right)}{\partial \theta}: \text { output sensitivity function } \\
\xi \quad \text { Marquardt parameter }
\end{array}\right.
$$

Output sensitivity functions can be computed by differentiating (17) with respect to $a_{i}, b_{i}, \gamma$.

The idea of optimizing the commensurable order instead of all differentiation orders was first introduced by Cois et al. [23] who chose to write the transfer function (17) in a modal form as in (8). They however constrained all $s^{\gamma}$-poles to be real-valued and of multiplicity one $\left(v_{k}=1, \quad \forall k\right)$. In general, $s^{\gamma}$-poles can be real or complex conjugate, and of multiplicity greater or equal to one. As a result, the following more restrictive class of models was proposed in [23] (see also figure (5)):

$$
F(s)=\sum_{k=1}^{N} \frac{A_{k}}{s^{\gamma}-s_{k}}
$$

The corresponding parameters' vector was then optimized:

$$
\theta^{\mathrm{T}}=\left[A_{1}, s_{1}, \cdots A_{N}, s_{N}, \gamma\right]
$$

In such a case, parameters are estimated recursively according to (34), the gradient and the hessian are computed using (35), and the sensitivity functions are obtained in a simpler form as:

$$
\begin{gathered}
\frac{\partial \hat{y}(t, \hat{\theta})}{\partial A_{k}}=\mathscr{L}^{-1}\left(\frac{1}{s^{\gamma}-s_{k}}\right) \otimes u(t) \\
\frac{\partial \hat{y}(t, \hat{\theta})}{\partial s_{k}}=\mathscr{L}^{-1}\left(\frac{A_{k}}{\left(s^{\gamma}-s_{k}\right)^{2}}\right) \otimes u(t)
\end{gathered}
$$

$$
\frac{\partial \hat{y}(t, \hat{\theta})}{\partial \gamma}=\mathscr{L}^{-1}\left(\sum_{k=1}^{N}-\frac{A_{k} s^{\gamma} \ln (s)}{\left(s^{\gamma}-s_{k}\right)^{2}}\right) \otimes u(t)
$$

Where $\otimes$ stands for convolution operator.

Note the presence of $\ln (s)$ in the last sensitivity function which makes analytical computation of the derivative complicated.

2.2.2 Method based on continuous-time simulation of fractional models Trigeassou et al. [13] take as a building block of fractional models a non integer integrator bounded in the frequency band as shown in (13) and described in section 1.5. The differentiation order is estimated by fixing $\alpha$ and computing $\eta$ of (13). Once $\alpha$ and $\eta$ known, the differentiation order $\gamma$ is deduced according to (14).

For the sake of simplicity, consider the following fractional differential system:

$$
\mathbf{D}^{\gamma} y(t)+a_{0} y(t)=b_{0} u(t)
$$

Define $X(s)$ as:

$$
X(s)=\frac{U(s)}{s^{n}+a_{0}}
$$

Thus, the generalized state-space representation of this system is obtained:

$$
\left\{\begin{array}{c}
\mathbf{D}^{\gamma} x(t)=-a_{0} x(t)+u(t) \\
y(t)=b_{0} x(t)
\end{array}\right.
$$

Equivalently, (43) can be written using continuous-time equivalent state space model (see section 1.5):

$$
\left\{\begin{array}{c}
\dot{x}_{1}(t)=-a_{0} x_{N+1}(t)+u(t) \\
y(t)=b_{0} x_{N+1}(t)
\end{array}\right.
$$

Taking into account the state space representation of the continuous-time approximation, the following global rational state space representation can be written:

$$
\left\{\begin{array}{c}
\dot{x}_{G}=A_{G} x_{G}+B_{G} u \\
y=C_{G} x_{G}
\end{array}\right.
$$


where:

$$
\begin{gathered}
A_{G}=\mathbf{A}+\left[\begin{array}{cccc}
0 & \cdots & 0 & -a_{0} \\
\vdots & \ddots & & 0 \\
\vdots & & \ddots & \vdots \\
0 & \cdots & 0 & 0
\end{array}\right] \\
B_{G}=\mathbf{B}, \quad C_{G}^{T}=\left[\begin{array}{llll}
0 & \ldots & 0 & b_{0}
\end{array}\right]
\end{gathered}
$$

$\mathbf{A}$ and $\mathbf{B}$ being defined in (15).

Identification algorithm In the case, the fractional behavior is limited in a frequency band, say $\left[\omega_{A}, \omega_{B}\right]$, then authors fix $\alpha$ and estimate the parameter vector:

$$
\theta^{T}=\left[a_{0}, b_{0}, \eta\right]
$$

In all cases the optimized criterion is defined as in (32) and (33). The coefficients are computed recursively according to (34) and (35). Sensitivity functions are now obtained by computing partial derivatives of (13) with respect to each of the parameter of (48).

2.2.3 Method based on orthogonal functions Recently, Malti et al. [16-18] have synthesized complete orthogonal basis in $L_{2}[0, \infty$ [ (Lebesgue space of squared integrable functions). Laguerre, Kautz, and GOB functions are hence extended to fractional differentiation orders. Thus, any finite energy stable transfer function $F(s)$ which belongs to the Hardy space $\mathrm{H}_{2}\left(\mathbb{C}^{+}\right)$ can be written as a linear combination of fractional orthogonal functions $G_{m}(s)$ :

$$
F(s)=\sum_{m=1}^{\infty} a_{m} G_{m}(s)
$$

Usually, (49) is truncated to a given order $N$ which is justified by the convergence of Fourier coefficients, $a_{m}$, as $m$ tends to infinity. $F(s)$ is hence approximated by the finite sum:

$$
F(s) \approx F_{M}(s)=\sum_{m=1}^{M} a_{m} G_{m}(s)
$$

Fractional basis are used in output error identification with fixed denominator models as described in [24]. Some key parameters need first of all to be fixed using prior knowledge such as commensurable order, Laguerre, kautz or all prescribed poles. Then, orthogonalization procedure is run according to the algorithms described in [16-18]. Finally, Fourier coefficients, $a_{m}$, which entirely define the system as in (50), are computed using least squares. The truncation order $N$ is fixed to obtain a satisfactory approximation. Akaike and Young information criteria can be used.

Assume $u(t), y(t), t \in[0, T]$ input and output data generated using a finite energy linear fractional model. The identification procedure consists of computing optimal coefficient vector $g=$ $\left[a_{1}, a_{2}, \ldots, a_{N}\right]^{T}$ which minimizes the least square error:

$$
J=\frac{1}{T} \int_{0}^{T}(\varepsilon(t))^{2} d t
$$

where

$$
\varepsilon(t)=y(t)-\sum_{m=1}^{M} a_{m} u_{G_{m}}(t)
$$

$y(t)$ and $u_{G_{m}(t)}$ are respectively the system and orthogonal network outputs:

$$
u_{G_{m}}(t)=G_{m}(t) \otimes u(t)
$$

Setting:

$$
\mathbf{u}_{\mathbf{G}}(t)=\left[u_{G_{1}}(t) u_{G_{2}}(t) \cdots u_{G_{M}}(t)\right],
$$

the optimum estimation of Fourier coefficients $\hat{\mathbf{g}}$ is given by the least squares formula:

$$
\hat{\mathbf{g}}=\left[\int_{0}^{T} \mathbf{u}_{\mathbf{G}}(t)^{T} \mathbf{u}_{\mathbf{G}}(t) d t\right]^{-1} \int_{0}^{T} \mathbf{u}_{\mathbf{G}}(t)^{T} y(t) d t
$$

All properties of least squares estimates (persistent excitation, variance on estimates) as stated in [25] apply in this context.

\section{Example}

To illustrate the use of fractional models in system identification, a semi-infinite dimensional thermal system is considered. It is constituted of a long aluminium rod heated by a resistor. To ensure unidirectional heat transfer, the entire surface of the rod is insulated. The temperature of the rod is measured at a distance $x=5 \mathrm{~mm}$ from the heated end (figure (6)). 


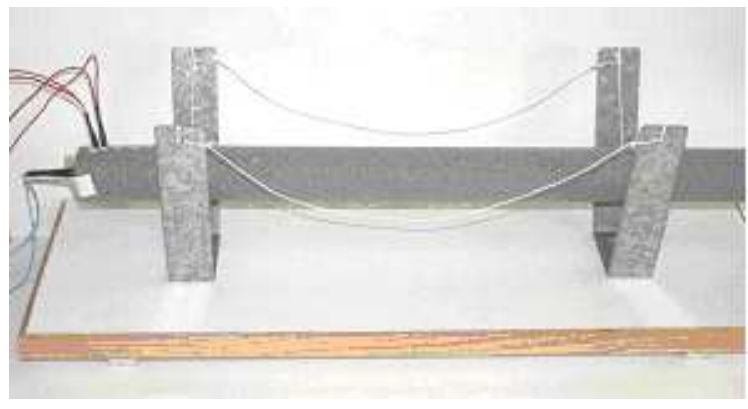

Figure 6. Insulated long aluminium rod heated by a resistor

The thermal system is considered as a semi-infinite plane homogenous medium initially at ambient temperature. Losses on the surface where the thermal flux is applied are neglected. Cois et al. [23] have shown that the analytical model linking the flux density applied on the outgoing normal surface of the medium to the temperature measured at an abscissa $x$ inside the medium has a commensurable order of 0.5 .

The thermal system is considered as a semi-infinite plane homogenous medium initially at ambient temperature. Losses on the surface where the thermal flux is applied are neglected. The model linking the flux density applied on the outgoing normal surface of the medium to the temperature measured at an abscissa $x$ inside the medium [3] is analytically given by:

$$
\tilde{H}(x, s)=\frac{T(x, s)}{\phi(x, s)}=\frac{1}{\sqrt{\lambda \rho C_{p}}} \frac{\sum_{k=0}^{\infty} a_{k}^{\prime} s^{\frac{k}{2}}}{\sum_{k=0}^{\infty}\left|a_{k}^{\prime}\right| s^{\frac{k+1}{2}}}
$$

with

$$
a_{k}^{\prime}=(-1)^{k} \frac{(x / 2)^{k}}{\alpha^{k / 2} k !}
$$

$\lambda$ and $\alpha$ denote the conductivity and the diffusivity of the medium. Relation (54) highlights that the thermal impedance of a semi-infinite medium is based on a commensurable order of 0.5 .

First of all, the system was identified by applying equationerror model and more precisely the SVF method. Identification data are plot on figure (7). The commensurable order was set to 0.5 and the following three parameters model was obtained:

$$
H_{1}(s)=\frac{0.256 s^{0.5}-0.002}{2.585 s^{1.5}+s}
$$

Then, equation error model was applied on the modal representation (36) and the commensurable order optimized. The
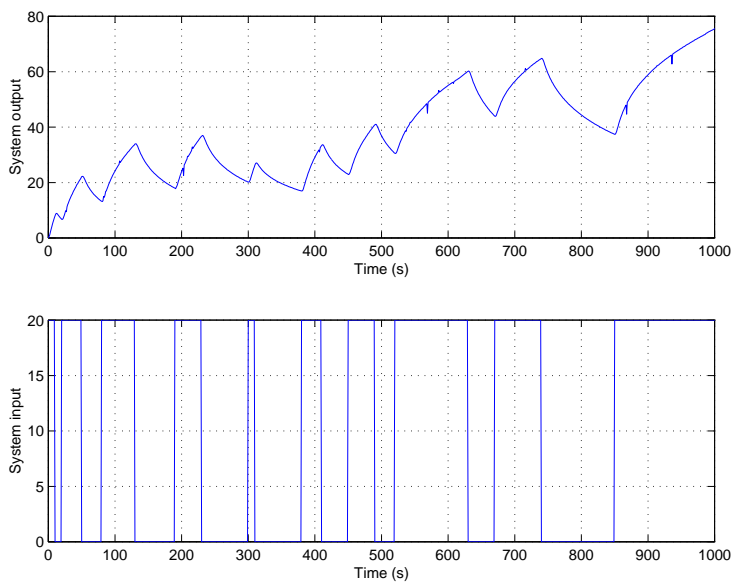

Figure 7. Estimation data

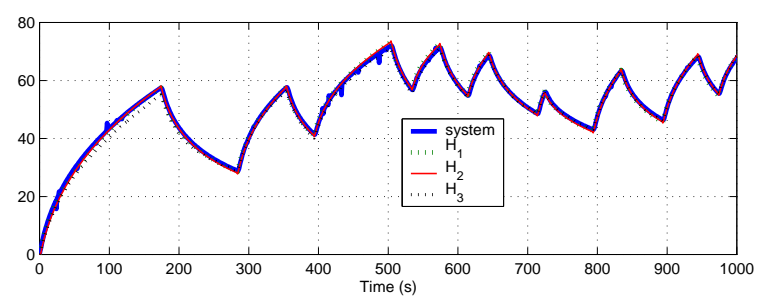

Figure 8. Validation data

following five parameters model was obtained:

$$
H_{2}(s)=\frac{0.319}{s^{0.483}+0.016}-\frac{0.530}{s^{0.483}+0.608}
$$

The optimal commensurable order is close to 0.5 as in the analytic model [23].

Next, for comparison purposes, a twelve parameters rational model was identified:

$$
\begin{aligned}
& H_{3}(s)= \\
& \quad \frac{-0.01 s^{5}+0.45 s^{4}-0.07 s^{3}+1.55 s^{2}+0.03 s+10^{-5}}{s^{6}+0.16 s^{5}+13.04 s^{4}+1.09 s^{3}+31.23 s^{2}+1.70 s+0.01}
\end{aligned}
$$

The normalized mean squared errors computed on validation data for both fractional models are close to each other: $\operatorname{NMSE}\left(\mathrm{H}_{1}\right) \approx \operatorname{NMSE}\left(\mathrm{H}_{2}\right) \approx 2 \times 10^{-4}$; whereas the normalized mean squared error of the rational model is: $\operatorname{NMSE}\left(\mathrm{H}_{3}\right) \approx$ $6 \times 10^{-4}$.

As shown on validation data of figure (8), the identified models give satisfactory results. 


\section{Conclusion and outlooks}

This paper presents a state of the art of actual achievements in time-domain system identification using fractional models. Mainly equation-error and output-error models are detailed. In the former, differentiation orders are fixed and only model's parameters are estimated. In the latter differentiation orders and model's coefficients can be estimated. One way for limiting the number of parameters consists of estimating the commensurable order and fixing all its multiples. For the time being, only white additive noise was considered. All model classes including colored noise should be extended to fractional differential orders. Moreover, system identification using stochastic signals is worth consideration.

Multiple other questions regarding fractional system identification remain unanswered. One of the most challenging is how to take into account initial conditions? This question cannot be answered as easily as in the rational case because a noninteger derivative of a signal depends on its whole past. Lorenzo et al. [26] showed that the effect of the past can be considered by taking into account an initialization function instead of a limited number of points. Can such a function be estimated?

\section{REFERENCES}

[1] Le Lay, L., 1998. "Identification fréquentielle et temporelle par modèle non entier". PhD thesis, Université Bordeaux I, Talence, France, Octobre.

[2] Lin, J., 2001. "Modélisatoin et identification de systèmes d'ordre non entier". PhD thesis, Université de Poitiers, France.

[3] Cois, O., 2002. "Systèmes linéaires non entiers et identification par modèle non entier : application en thermique". $\mathrm{PhD}$ thesis, Université Bordeaux 1, Talence, France.

[4] Aoun, M., 2005. "Systèmes linéaires non entiers et identification par bases orthogonales non entières". PhD thesis, Université Bordeaux 1, Talence, France, October 21st.

[5] Battaglia, J.-L., Le Lay, L., J.-C., B., A., O., and O., C., 2000. "Heat flux estimation through inverted non integer identification models". Int. J. of Thermal Science, 39(3), pp. 374-389.

[6] Darling, R., and Newman, J., 1997. "On the short behavior of porous intercalation electrodes". J. Electrochem. Soc., 144(9), pp. 3057-3063.

[7] Ortigueira, M., and Tenreiro Machado, J., eds., 2006. Signal Processing - special issue: Fractional calculus applications in signals and systems, Vol. 86. Elsevier, October.

[8] Samko, S., Kilbas, A., and Marichev, O., 1993. Fractional integrals and derivatives: theory and applications. Gordon and Breach Science.

[9] Grünwald, A., 1867. "Ueber begrenzte derivationen und deren anwendung". Zeitschrift für Mathematik und Physik, pp. 441-480.
[10] Oldham, K., and Spanier, J., 1974. The fractionnal calculus. Academic Press, New-York and London.

[11] Matignon, D., 1994. "Représentations en variables d'état de modèles de guides d'ondes avec dérivation fractionnaire". PhD thesis, Université de Paris-Sud, Orsay.

[12] Oustaloup, A., 1995. La dérivation non-entière. Hermès Paris.

[13] Trigeassou, J.-C., Poinot, T., Lin, J., Oustaloup, A., and Levron, F., 1999. "Modeling and identification of a non integer order system". In ECC.

[14] Matignon, D., 1998. "Stability properties for generalized fractional differential systems". ESAIM proceedings Systèmes Différentiels Fractionnaires - Modèles, Méthodes et Applications, $\mathbf{5}$.

[15] Malti, R., Aoun, M., Cois, O., and Oustaloup, A., 2003. "H2 norm of fractional differential systems". In ASME'03, Vol. DETC2003/VIB-48387.

[16] Malti, R., Aoun, M., Levron, F., and Oustaloup, A., 2005. Fractional Differentiation and its Applications. Vol. 1 - Mathematical tools, geometrical and physical aspects. $\mathrm{ch}$. Unified construction of fractional generalized orthogonal bases, pp. 87-102.

[17] Aoun, M., Malti, R., and Oustaloup, A., 2005. "Synthesis and simulation of fractional orthonormal bases". In 16th World IFAC Congress, IFAC, Elsevier.

[18] Aoun, M., Malti, R., Levron, F., and Oustaloup, A., 2007. "Synthesis of fractional Laguerre basis for system approximation”. Automatica, Preprints.

[19] Oustaloup, A., 1983. Systèmes asservis linéaires d'ordre fractionnaire. Masson - Paris.

[20] Cois, O., Oustaloup, A., Poinot, T., and Battaglia, J.-L., 2001. "Fractional statevariable filter for system identification by fractional model". In IEEE 6th European Control Conference (ECC'2001).

[21] Young, P., 1981. "Parameter estimation for continuous-time models - a survey". Automatica, 17(1), pp. 23-29.

[22] Marquardt, D., 1963. "An algorithm for least-squares estimation of non-linear parameters". J. Soc. Industr. Appl. Math., 11(2), pp. 431-441.

[23] Cois, O., Oustaloup, A., Battaglia, E., and Battaglia, J.-L., 2000. "Non integer model from modal decomposition for time domain system identification". In proceedings of 12th IFAC Symposium on System Identification, SYSID.

[24] Wahlberg, B., 1991. "System identification using Laguerre models". IEEE TAC, 36, pp. 551-562.

[25] Ljung, L., 1999. System identification-Theory for the user, 2 ed. Prentice-Hall, January.

[26] Lorenzo, C., and Hartley, T., 2000. "Initialized fractional calculus". Int. J. of Applied Mathematics, 3(3), September, pp. 249-266. 